\title{
B. LEMAIRE
}

\section{Brève communication. Théorème de conservation des clients dans les files d'attente}

Revue française d'automatique, d'informatique et de recherche opérationnelle. Recherche opérationnelle, tome 12, no 4 (1978), p. 395-399.

<http://www.numdam.org/item?id=RO_1978_12_4_395_0>

(C) AFCET, 1978, tous droits réservés.

L'accès aux archives de la revue « Revue française d'automatique, d'informatique et de recherche opérationnelle. Recherche opérationnelle » implique l'accord avec les conditions générales d'utilisation (http://www.numdam.org/ legal.php). Toute utilisation commerciale ou impression systématique est constitutive d'une infraction pénale. Toute copie ou impression de ce fichier doit contenir la présente mention de copyright.

\section{Numdam}

Article numérisé dans le cadre du programme

Numérisation de documents anciens mathématiques

http://www.numdam.org/ 


\title{
Brève communication
}

\section{THÉORẼME DE CONSERVATION Des Clients dANS LeS Files D'ATtente (*)}

\author{
par B. Lemaire $\left({ }^{1}\right)$
}

\begin{abstract}
Résumé. - Dans cette note, on démontre le théorème de conservation des clients pour un système en régime permanent à un seul guichet, dans un cas très général (lois d'arrivées et de services à transformées de Laplace rationnelles), en ramenant l'étude à celle d'un processus markovien auquel on applique le théorème de conservation du fux de transition.
\end{abstract}

\section{NOTATIONS ET RÉSULTATS PRÉLIMINAIRES}

Pour toute file d'attente en régime permanent, le taux des entrées dans le système est égal, intuitivement, à celui des sorties puisqu'il ne s'y produit ni accumulation, ni disparition, ni génération spontanée de clients. C'est ce que nous démontrons ici pour un système à guichet unique et file d'attente de taille illimitée. On suppose que le régime permanent est atteint et, en outre, que les transformées de Laplace des densités de probabilité des inter-arrivées (intervalles de temps séparant deux arrivées consécutives) et des services sont rationnelles : c'est, en particulier, le cas de lois exponentielles, d'Erlang ou encore hyperexponentielles. Lorsque la densité de probabilité $s(t)$ de la durée des services admet une transformée de Laplace $S^{*}(u)$ rationnelle, on peut simuler cette loi de service - comme l'a démontré Cox [1] - par un réseau de guichets fictifs à service exponentiel, appelé « zone de service » (z. s.), toute z. s. servant, au plus, un client à la fois (fig. 1):

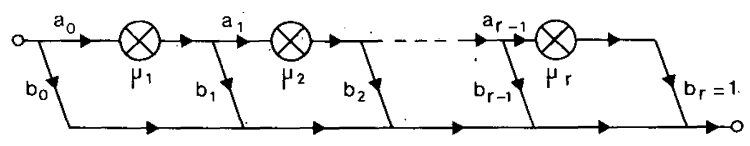

Figure 1

(*) Manuscrit reçu Mars 1978.

(') Laboratoire de Recherche Opérationnelle, C.N.A.M., Paris.

R.A.I.R.O. Recherche opérationnelle/Operations Research, vol. 12, $\mathrm{n}^{\circ} 4$, novembre 1978 
Dans une z. s., $a_{i}$ (resp. $b_{i}$ ) désigne la probabilité pour qu'un client se rende au $(i+1)$-ième guichet après être passé au $i$-ième (resp. quitte le système); on a $: a_{i}+b_{i}=1$. Les probabilités de branchement $a_{i}$ et $b_{i}$ peuvent être complexes, de même que les taux de services $\mu_{i}$ des guichets fictifs et les calculs peuvent être menés formellement [1].

Comme :

$$
S^{*}(u)=b_{0}+\sum_{i=1}^{r} a_{0} a_{1} \ldots a_{i-1} b_{i} \prod_{j=1}^{i} \frac{\mu_{j}}{u+\mu_{j}},
$$

les taux $\mu_{j}$ ne sont autres, au signe près, que les pôles de $S^{*}(u)$, les probabilités $a_{i}$ et $b_{i}$ s'obtenant par identification.

Nous considérerons plus généralement, des réseaux de guichets fictifs représentés par un graphe connexe, sans circuit, comportant une seule entrée et une seule sortie; ces derniers, dont le type Cox est un cas particulier, seront dits de type $\mathscr{R}$.

Il arrive qu'un réseau de type $\mathscr{R}$, avec des probabilités de branchement réelles, puisse être substitué à un réseau de Cox à probabilités complexes.

Symétriquement, une loi d'arrivée à transformée de Laplace rationnelle peut être simulée par un réseau de Cox (les $\lambda_{i}, \alpha_{i}, \beta_{i}$ correspondant resp. aux $\mu_{i}, a_{i}, b_{i}$ ) ou bien par un réseau du type $\mathscr{R}$, qui sera appelé " générateur d'arrivées » (g. a.). Le g. a. est alimenté par un réservoir infini de clients; à l'intérieur du g. a. se trouve, à tout instant, un client et un seul.

\section{PROCESSUS DE MARYOV ASSOCIE}

Lorsque les lois de services et d'arrivées sont simulées par un réseau de Cox ou de type $\mathscr{R}$, on peut associer un processus de Markov au phénomène étudié ; les états $E_{l, n, m}$ du processus sont tels que :

$l$ désigne le numéro du guichet occupé du g. a. : $l \in L=\{1,2, \ldots, k\}$;

$n$ désigne le nombre de clients présents dans le système (file d'attente et z. s., à l'exclusion du g. a.);

$m$ désigne le numéro du guichet occupé de la z. s. : $m \in M=\{1, \ldots, r\} \cup\{0\}$ (par convention $m=0$ si le système est vide).

Les durées de séjour aux guichets fictifs du g. a. et de la z. s. étant des variables aléatoires exponentielles, donc "sans mémoire ", le comportement du système est markovien: les probabilités correspondant aux différents états à $t+h$ ne dépendent que de l'état du système à $t$, sans qu'il soit nécessaire de connaître l'évolution du système entre 0 et $t$.

R.A.I.R.O. Recherche opérationnelle/Operations Research 
Notons $\beta_{i}$ (resp. $b_{i}$ ) la probabilité pour qu'un client, une fois son séjour terminé au $i$-ième guichet du g. a. (resp. de la z. s.) pénètre dans (resp. quitte) le système.

\section{APPLICATION DU THÉORÈME DE CONSERVATION DU FLUX DE TRAN- SITION}

Désignons par $C_{N}$ l'ensemble des états pour lesquels $N$ clients au plus sont présents dans le système : $C_{N}=\left\{E_{l, n, m} \mid l \in L, n \leqslant N, m \in M\right\}$. Le théorème de conservation du flux de transition [3], [4] énonce que, pour tout processus de Markov homogène ergodique, d'ensemble d'états $X$, et pour tout sousensemble $A \subset X$, en régime permanent, la fréquence des transitions vers l'extérieur de $A$ (c'est-à-dire des états de $A$ vers ceux de $X-A$ ) égale la fréquence des transitions vers l'intérieur de $A$ :

$$
\forall A \subset X: \quad \sum_{i \in A} \sum_{j \in X-A} p_{i} \alpha_{i j}=\sum_{j \in X-A} \sum_{i \in A} p_{i} \alpha_{j i},
$$

où : $\alpha_{i j} . d t+o(d t)$ est la probabilité élémentaire de transition, entre $t$ et $t+d t$, de l'état $E_{i}$ vers l'état $E_{j}$.

En prenant $C_{N}$ comme sous-ensemble $A$, l'égalité des fréquences de transition s'écrit :

$$
\sum_{\substack{l \in L \\ m \in M}} \lambda_{l} \cdot \beta_{l} \cdot p_{l, N, m}=\sum_{\substack{l \in L \\ m \in M}} \mu_{m} \cdot b_{m} \cdot p_{l, N+1, m}
$$

En effet, les seuls arcs traversant $C_{N}$ sont :

Dans un sens, les arcs associés aux transitions d'états pour lesquels $N$ clients sont présents vers des états pour lesquels $N+1$ clients le sont : elles correspondent à l'arrivée d'un client dans le système, à partir des états $E_{l, N, m}(l \in L$, $m \in M)$ c'est-à-dire à la sortie d'un client du g. a. qui se trouvait en un guichet fictif $l$ de taux $\lambda_{l}(l \in L)$, y a terminé son service, et ensuite est sorti directement (avec la probabilité $\beta_{l}$ ). La fréquence totale de ces transitions s'obtient en sommant sur $l$ et $m$ les quantités $\lambda_{l} \cdot \beta_{l} \cdot p_{l, N, m}$.

En sens inverse, les arcs correspondent aux transitions d'états pour lesquels on passe de $N+1$ à $N$ clients présents : il faut donc, qu'à partir des états $E_{l, N+1, m}(l \in L, m \in M)$, il y ait eu une sortie du système ce qui n'est possible que si le client de la z. s. (qui n'est pas vide) se trouvant au guichet fictif $m$ de taux $\mu_{m}(m \in M)$, a terminé son service, et ensuite est sorti directement (avec la probabilité $b_{m}$ ). La fréquence totale de ces transitions s'obtient en sommant sur $l$ et $m$ les quantités $\mu_{m} b_{m} p_{l, N+1, m}$.

vol. $12, \mathrm{n}^{\circ} 4$, novembre 1978 


\section{Posons :}

$$
\begin{array}{ll}
\pi_{l}=\sum_{\substack{N \in \mathbf{N} \\
m \in M}} p_{l, \dot{N}, m} ; \quad \pi_{l} \text { représente la probabilité pour que le } l \text {-ième guichet du } \\
\text { g. a. soit occupé ( } \mathbf{N} \text { désigne l'ensemble des entiers); }
\end{array}
$$
et :

$Q_{m}=\sum_{\substack{N \in N \\ l \in L}} p_{l, N+1, m} ; \underset{\text { de la } \text { z. s. soit occupé. }}{Q_{m} \text { représente la probabilité pour que le } m \text {-ième guichet }}$

La sommation membre à membre des relations $(E)$ pour $N=0,1, \ldots$ fournit :

$$
\sum_{l \in L} \lambda_{l} \cdot \beta_{l} \cdot \pi_{l}=\sum_{m \in M} \mu_{m} \cdot b_{m} \cdot Q_{m} .
$$

a) Soit $\gamma_{l}$ la probabilité pour que les clients passent par le guichet $l$ du g. a. (pour le réseau de Cox : $\gamma_{l}=\alpha_{0} \alpha_{1} \ldots \alpha_{l-1}$ ). $\pi_{l}$ est aussi le quotient de l'espérance du temps passé au guichet $l$ qui vaut $\gamma_{l} / \lambda_{l}$, par l'espérance du temps de séjour dans le générateur qui vaut $\frac{1}{\lambda}$ :

$$
\pi_{l}=\frac{\gamma_{l} / \lambda_{l}}{\sum_{l \in L} \gamma_{l} / \lambda_{l}}=\gamma_{l} \cdot\left(\lambda / \lambda_{l}\right)
$$

L'événement « tout client sortira du générateur » étant certain, on a :

D'où :

$$
\sum_{l \in L} \gamma_{l} \cdot \beta_{l}=1
$$

$$
\sum_{l \in L} \lambda_{i} \cdot \beta_{l} \cdot \pi_{i}=\lambda \sum_{l \in L} \beta_{i} \cdot \gamma_{l}=\lambda
$$

Ainsi le premier membre de $(F)$ est égal à $\lambda$.

b) Le taux moyen du service vaut $\mu$ et la probabilité pour que le système soit vide : $p_{0}=\sum_{l \in L} p_{l, 0,0}$; soit $c_{m}$ la probabilité pour que les clients passent par le guichet $m$ de la z. $\mathrm{s}$. (pour le réseau de $\operatorname{Cox}: c_{m}=a_{0} . a_{1} \ldots a_{m-1}$ ). Notons que la z. s. n'est pas utilisée en permanence, comme l'est le g. a. La probabilité $\hat{Q}_{m}$ pour que le guichet $m$ soit gccupé, lorsque la $\mathrm{z}$. s. n'est pas vide de client, est telle que :

$$
\hat{Q}_{m}=\frac{c_{m} / \mu_{m}}{\sum_{m \in M} c_{m} / \mu_{m}}=c_{m} \cdot\left(\mu / \mu_{m}\right), \quad \text { car } \quad \frac{1}{\mu}=\sum_{m \in M} \frac{c_{m}}{\mu_{m}}
$$

Par application du théorème des probabilités totales :

$$
Q_{m}=\hat{Q}_{m}\left(1-p_{0}\right)+0 \cdot p_{0} \quad \text { d'où }: Q_{m}=\left(1-p_{0}\right) \cdot c_{m} \cdot\left(\mu / \mu_{m}\right)
$$


L'événement « tout client sortira de la z. s. " étant certain, on a :

D'où :

$$
\sum_{m \in M} c_{m} \cdot b_{m}=1
$$

Finalement :

$$
\sum_{m \in M} \mu_{m} \cdot b_{m} \cdot Q_{m}=\mu\left(1-p_{0}\right) \sum_{m \in M} c_{m} \cdot b_{m}=\mu\left(1-p_{0}\right)
$$

$$
\lambda=\mu\left(1-p_{0}\right)
$$

Théorème : Le taux $\lambda$ des arrivées des clients dans le système considéré égale celui des sorties des clients, soit $\mu\left(1-p_{0}\right)$, produit du taux de service $\mu$ par la probabilité $\left(1-p_{0}\right)$ que le guichet soit actif.

On peut démontrer également la conservation des clients en chaque guichet fictif de la z. s. On obtient, en effet $: \mu_{m} \cdot Q_{m}=\lambda . c_{m}$ en remplaçant $1-p_{0}$ $\operatorname{par} \frac{\lambda}{\mu}$ :

Le taux des entrées au guichet $m$ (produit du taux des arrivées $\lambda$ par la probabilité de passer par ce guichet), égale le taux des sorties de ce même guichet, qui n'est autre que le produit de son taux de service $\mu_{m}$ par la probabilité $Q_{m}$ qu'il soit actif.

On obtient un résultat analogue pour le g. a., puisque : $\lambda_{l} \cdot \pi_{l}=\lambda \cdot \gamma_{l}$.

\section{BIBLIOGRAPHIE}

1. D. R. Cox, A use of complex probabilities in the theory of stochastic processes, Proceedings of Cambridge Philosophical Society, 51, 1955, p. 313-319.

2. M. KRAKowsKI, Conservation Methods in queuing theory. R.A.I.R.O., série verte, vol. 1, 1973, p. 63-83.

3. B. Lemaire, Méthode de conservation et blocage dans les files d'attente. R.A.I.R.O., série verte, vol. 4, 1977, p. 363-377.

4. B. Lemaire, Contribution à l'étude des systèmes avec attente: les méthodes de conservation. Application à des problèmes de recherche opérationnelle et d'informatique, thèse de Doctorat d'État, Université Pierre et Marie Curie (Paris VI), juin 1978. 